\title{
BMC Pregnancy and Childbirth reviewer acknowledgement 2014
}

Nawsheen Boodhun

\section{Contributing reviewers}

The editors of BMC Pregnancy and Childbirth would like to thank all our reviewers who have contributed to the journal in Volume 14 (2014).

Vigdis Aasheim

Norway

Edgardo Abalos

Argentina

Parvin Abedi

Iran

Peter Abrahams

UK

Barbara Abrams

USA

Ishag Adam

Sudan

Babatunde Adedokun

Nigeria

Mulat Adefris

Ethiopia

Ramesh Adhikari

Nepal

Asri Adisasmita

Indonesia

Werner Adler

Germany

Philip Adongo

Ghana

Abdallah Adra

Lebanon
Dinesh Agarwal
India

Mukesh Mansha Agarwal

United Arab Emirates

Schadrac Agbla

Benin

Kingsley Agho

Australia

Priya Agrawal

USA

Regina Aguiar

Brazil

Charles Agyemang

Netherlands

Jamil Ahmed

Pakistan

Saifuddin Ahmed

USA

Noori Akhtar-Danesh

Canada

Stella Akinleye

Nigeria

Olufunke Alaba

South Africa

Ashraful (Neeloy) Alam

Australia
Salvatore Alberico

Italy

Juan Luis Alcazar

Spain

Sophie Alexander

Belgium

Charles Algert

Australia

Victoria Allen

Canada

Nedal Alnawaiseh

Jordan

Charles Ameh

UK

Richard Amenyah

Burkina Faso

Amanda Ampt

Australia

Sarah Amugongo

Kenya

Judith Anchang-Kimbi

Cameroon

Anne-Marie Nybo Andersen Denmark

Kathryn Andersen

USA

Correspondence: Nawsheen.Boodhun@biomedcentral.com

BioMed Central, Floor 6, 236 Gray's Inn Road, London WC1X 8HB, UK 
Cynthie Anderson
USA

Nasratullah Ansari

Afghanistan

Marcela Agustina Araya Bannout

Chile

Brigid Arkins

Ireland

Raul Artal

USA

Atnafu Getachew Asfaw

Ethiopia

Catherine Ashwin

UK

Nega Assefa

Ethiopia

Lou Atkinson

UK

Laura Attanasio

USA

Ingvild Aune

Norway

Marie-Paule Austin

Australia

Francisco Avila

Chile

Jennifer Ayton

Australia

Hassan Ba'Aqeel

Saudi Arabia

Frank Baarveld

Netherlands

Rashmi Bagga

India

Patricia Bailey

USA

Lesley Baillie

USA

Emily Bain

Australia

Kathleen Baird

Australia

Murat Bakacak

Turkey
Marian Bakker

Netherlands

Wouter Bakker

Netherlands

Marie-Clare Balaam

UK

Tatiana Balachova

USA

Subha Sri Balakrishnan

India

Julius Bamidele

Nigeria

Germana Bancone

Thailand

Sunita Bandewar

Canada

Melanie Bannister-Tyrrell

Australia

Flora Maria Barbosa Da Silva

Brazil

Lesley Barclay

Australia

Madeleine Barnett

UK

Mauro Barni

Italy

Hyam Bashour

Syria

Sriparna Basu

India

B Bateman

USA

Linda Bauld

UK

Hamideh Bayrampour

Canada

Eva Bazant

USA

Sarah Beake

UK

Roxanne Beauclair

South Africa

Bérengère Beauquier-Maccotta

France
Helen Bedford

UK

Carol Bedwell

UK

Katrien Beeckman

Belgium

Andrea Begley

Australia

Yaakov Beilin

USA

Mesfin Awoke Bekalu

Ethiopia

Mireille Bekker

Netherlands

Mandy Bell

USA

Katrien Benhalima

Belgium

Karen Benzies

Canada

Marie Berg

Sweden

Annette Bernloehr

Germany

Peter Berti

Canada

Miteshkumar Bhanderi

India

Divya Kanwar Bhati

India

Sohinee Bhattacharya

UK

Yagya Bhurtyal

UK

Debra Bick

UK

Cara Bicking Kinsey

USA

Lorena Binfa

Chile

Connie Bish

USA

Antje Blank

Germany 
Hannah Blencowe

UK

Ellen Blix

Norway

Paula Bolton-Maggs

UK

Jermane Bond

USA

Marie-Pierre Bonnet

France

Elizabeth Bonney

USA

Matteo Bonomo

Italy

Matteo Bonzini

Italy

Matthias Borchert

Germany

Georgios Bouras

Greece

Marielle Karine Bouyou-Akotet

Gabon

Angela Bowen

Canada

Carol Bower

Australia

Katherine Bowers

USA

Elaine Boyle

UK

Marek Brabec

Czech Republic

Loretta Brabin

UK

Carmel Bradshaw

Ireland

Petra Brhlikova

UK

Annette Briley

UK

Carinne Brody

USA

Sharon Bruce

Canada
Sherri Bucher

USA

Eckhart Buchmann

South Africa

Henna Budhwani

USA

Irina Buhimschi

USA

Anne Buist

Australia

Maria Bullarbo

Sweden

Joanna Busza

UK

Elizabeth Butrick

USA

Alexander Butwick

USA

Abbey Byrne

Australia

Joanne Cacciatore

USA

Jennifer Callaghan-Koru

USA

Wendy Camelo Castillo

USA

Christina Campbell

USA

Angela Carberry

Australia

Michael Carson

USA

John Carstensen

Sweden

Christine Catling-Paull

Australia

Ricardo Cavalli

Brazil

Juraci Cesar

Brazil

Dong Hyun Cha

Korea South

Christina Chambers

USA
Ronna Chan

USA

Yi-Sheng Chao

Canada

Lucy Chappell

UK

Lisa Chasan-Taber

USA

Sarika Chaturvedi

India

Picklu Chaudhuri

India

Phaikyeong Cheah

Thailand

Yingyao Chen

China

Li-Yin Chien

Taiwan

Karmel Choi

USA

Abdul Mannan Choudhury

Bangladesh

Ole Bjarne Christiansen

Denmark

Cynthia Chuang

USA

John Cleland

UK

Tim Colbourn

UK

John Condon

Australia

Christine Connors

Australia

Stephen Contag

USA

Martina Cornel

Netherlands

Giuliana Cortese

Italy

Maria Laura Costa

Brazil

Karen Cowgill

USA 
Denis Crankshaw

Canada

Debra Creedy

Australia

Jenny Cresswell

UK

David Cromwell

UK

Doug Cronie

Netherlands

Rhonda Curran

UK

Andrew E. Czeizel

Hungary

Fabricio Da Silva Costa

Australia

Per Damkier

Denmark

Cem Dane

Turkey

Francesco D'Antonio

Italy

Zoe Darwin

UK

Sandra David Tchouda

France

Michael Davies

Australia

Deborah Davis

Australia

Angela Dawson

Australia

Vincent De Brouwere

Belgium

Gaia De Campora

Italy

Johanna De Graaf

Netherlands

Claire De Labrusse

Switzerland

Francine De Montigny

Canada

Paul De Reu

Netherlands
Eugene Declercq

USA

Tedbabe Degefie

Ethiopia

Stephanie Dellicour

UK

Stephanie Delong

USA

Anna Dencker

Sweden

Barbara Dennison

USA

Costanzo Di Maria

UK

Mirko Di Martino

Italy

Nadia Diamond-Smith

USA

Cyril Dim

Norway

Cyril Dim

Nigeria

Jodie Dionne-Odom

USA

Khady Diouf

USA

Mai Do

USA

Jodie Dodd

Australia

Edel Doherty

Ireland

Sara Donahue

USA

Amol Dongre

India

Soo Downe

UK

Michele Drehmer

Brazil

Mari Dumbaugh

Switzerland

Corinne Dupont

France
Jill Durocher

USA

Elizabeth Duthie

USA

Indranil Dutta

India

Johannes Duvekot

Netherlands

Alexandra Duxbury

UK

Els Duysburgh

Belgium

Pratibha Dwarkanath

India

Michelle Dynes

USA

Christine East

Australia

Thomas Ebert

Germany

Winifred Eboh

UK

Elizabeth Echoka

Kenya

Michael Ediau

Uganda

Roger Edwards

USA

Bolaji Egbewale

Nigeria

Anne Ego

France

Samantha Ehrlich

USA

Kristjana Einarsdottir

Australia

Maria Ekelin

Sweden

Alison El Ayadi

USA

Julio Elito Jr.

Brazil

Waleed El-Khayat

Egypt 
Sohier Elneil

UK

Susheela Engelbrecht

USA

Cyril Engmann

USA

Offer Erez

Israel

Jan Jaap H.M. Erwich

Netherlands

Olapeju Esimai

Nigeria

Michael Esplin

USA

Birgitta Essén

Sweden

Holly Essex

UK

Titilayo Fakeye

Nigeria

Shangrong Fan

China

Sara Farchi

Italy

Marcelo Farias

Chile

Bukola Fawole

Nigeria

Maya Fehling

Germany

Marlena Fejzo

USA

Gedefaw Abeje Fekadu

Ethiopia

Tanis Fenton

Canada

Jennifer Fenwick

Australia

Leonides Fernandez

Spain

Enrico Ferrazzi

Italy

Catherine Fetherston

Australia
Tamara Fetters

USA

Francesc Figueras

Spain

Kirstin Finning

UK

Jane Fisher

Australia

Mirembe Florenc

Uganda

Andrew Fogarty

UK

Bruno M Fonseca

Portugal

Jane Ford

Australia

Liz Forty

UK

Jean Christophe Fotso

Kenya

Maralyn Foureur

Australia

Mirjam Fransen

Netherlands

Jennifer Fraser

Australia

Heather Frey

USA

Yechiel Friedlander

Israel

J. Frederik Froen

Norway

Suthat Fucharoen

Thailand

Judith Fullerton

USA

Anita Gagnon

Canada

Jenny Gamble

Australia

Rebecca Ganann

Canada

Robin Gandley

USA
Marie Gantz

USA

Ana Garces

Guatemala

Deepak Garg

UK

Susan Garthus-Niegel

Norway

Deirdre Gartland

Australia

Asheber Gaym

Ethiopia

Abebaw Gebeyehu

Ethiopia

Yirgu Gebrehiwot

Ethiopia

Alem Gebremariam

Ethiopia

Samson Gebremedhin

Ethiopia

Diederike Geelhoed

Mozambique

Ado Geidam

Nigeria

Caitlin Gerdts

USA

Rebecca Giallo

Australia

Frances Gibson

Australia

Kelly Gibson

USA

Melanie Gibson-Helm

Australia

Emma L Giles

UK

Katie Gillies

UK

Erik Giltay

Netherlands

Sarah Gimbel

USA

Yehuda Ginosar

Israel 
Beatriz F. Giraldo

Spain

Fiseha Girma

Ethiopia

Carmen Giurgescu

USA

Salvatore Gizzo

Italy

Svetlana Glinianaia

UK

Marewa Patricia Glover

New Zealand

Katherine Goetzinger

USA

Srinivas Goli

India

Gustavo Gonzales

Peru

Adrienne Gordon

Australia

Michael Gravett

USA

Richard Griffey

USA

Malcolm Griffiths

UK

Peta Grigsby

USA

Rosalie Grivell

Australia

Karin Gross

Switzerland

Pietro Grussu

Italy

Susanne Grylka-Baeschlin

Germany

Ruth Guinsburg

Brazil

Marie-Julia Guittier

Switzerland

Anil Gumber

UK

Freedom Nkhululeko Gumedze

South Africa
Ricardo Gurgel

Brazil

Ipek Gurol-Urganci

UK

Muhammad Atif Habib

Australia

Dereje Habte

Botswana

Eleni Hadjigeorgiou

Cyprus

Jeannie Haggerty

Canada

Demewoz Haile

Ethiopia

Desta Hailu

Ethiopia

Arja Halkoaho

Finland

Wendy Hall

Canada

Stephen Halpern

Canada

Mohga Hammad

Egypt

Karin Hammarberg

Australia

Gillian Hanley

Canada

Tharangrut Hanprasertpong

Thailand

Claudia Hanson

Sweden

Jessica Hanson

USA

Philip Harber

USA

Susanne Harder

Denmark

Lorie Harper

USA

Junichi Hasegawa

Japan

Mahmoud Hassan

France
Hossein Hassani

UK

Elizabeth Hatch

USA

Abigail Hatcher

South Africa

Marie Hatem

Canada

Margaretha Haugen

Norway

T Lee-Ann Hawkins

Canada

Louise Hayes

UK

Maureen Heaman

Canada

Alexander Heazell

UK

Steffie Heemelaar

Netherlands

Rebecca Helmreich

USA

Jane Henderson

UK

Jennifer Henderson

Australia

Amanda Henry

Australia

Edgar Hernandez Andrade

USA

Florian Herse

Germany

Nicola Heslehurst

UK

Philip Hess

USA

Jenny Hewison

UK

Briony Hill

Australia

Zelee Hill

UK

Lisa Hinton

UK 
Janet Hirst

UK

\section{Abraham Hodgson \\ Ghana \\ Hans V Hogerzeil \\ Netherlands}

Anna-Clara Hollander

Sweden

Jennifer Hollowell

UK

Sara Holton

Australia

Caroline Homer

Australia

Claire Homeyard

UK

Jaco Homsy

USA

Dell Horey

Australia

Shigeko Horiuchi

Japan

Gerard Hornstra

Netherlands

Jaime Horton

USA

John Horwood

New Zealand

Nazli Hossain

Pakistan

Sennen Hounton

Burkina Faso

Wendy Hoy

Australia

Amber Hromi-Fiedler

USA

Daniel Hruschka

USA

Kun Huang

China

Carl Hubel

USA

Megan Huchko

USA
Thomas Hulsey

USA

Tracy Humphrey

UK

\section{Tai-Ho Hung}

Taiwan

Jon Hyett

Australia

Lawrence Ikamari

Kenya

Jane Iles

UK

Lawrence Impey

UK

Victor Inem

Nigeria

Bilal Iqbal Avan

UK

Por Ir

Cambodia

Chibuike Iruloh

UK

M Mazharul Islam

Oman

Aditi Iyer

India

Nicola Jackson

Thailand

Laurie James-Hawkins

USA

Elisabeth Jangsten

Sweden

Patricia Janssen

Canada

Pouya Javadian

USA

Larissa Jennings

USA

Esther Jimenez

Spain

Helene Johns

Australia

Kate Jolly

UK
Julie Jomeen

UK

Catriona Jones

UK

Sasi Jonnalagadda

USA

Ruby Jose

India

K Joseph

Canada

Chandni Joshi

Australia

Racape Judith

Belgium

Othman Kakaire

Uganda

Douglas Kalman

USA

Christabel Kambala

Germany

Ahmed Kamel Eldesouky

Egypt

Martin Kammerer

UK

Lucy Kanya

Kenya

Marianna Karachaliou

Greece

Rajendra Karkee

Nepal

Fyson Kasenga

Malawi

Noriko Kato

Japan

Bekana Kebede

Ethiopia

William Keenan

USA

Susan Kelly

UK

Shona Kelly

UK

Bryn Kemp

UK 
Tamil Kendall
USA

Holly Kennedy

USA

Sara Kenyon

UK

Rosemary Keogh

Australia

Michail Keramidas

Sweden

Asma Khalil
UK

Monir Khalil

Libya

Attia Khan

Pakistan

Michelle Khan

USA

Shane Khan

USA

Vishnu Khanal

Nepal

Soghra Khani

Iran

Sunil Khanna

USA

Ali S Khashan

Ireland

Sharon Kibwana

Ethiopia

Hussein Lesio Kidanto

Tanzania

Masahiro Kihara

Japan

Deborah Kim

USA

Jo Kim

USA

Jinseok Kim

Korea South

Elizabeth Kimani-Murage

Kenya

Carol Kingdon

UK
Dawn Kingston

Canada

Brooke Kinniburgh

Canada

Tarja I Kinnunen

Finland

Russell Kirby

USA

Pertti Kirkinen

Finland

Sharon Kirkpatrick

Canada

Dmitry Kissin

USA

Kristen Kjerulff

USA

Michael Klein

Canada

Atle Klovning

Norway

Hannah Knight

UK

Marian Knight

UK

Thecla Kohi

Tanzania

Theano Kokkinaki

Greece

Owonikoko Kola

Nigeria

Klaas Koop

Netherlands

Tomomi Kotani

Japan

Mirjana Kovac

Serbia

Katy Kozhimannil

USA

Naoko Kozuki

USA

Karoline Kragelund Nielsen

Denmark

Gv Krishnaveni

India
Carsten Krüger

Germany

Andrzej Kulczycki

USA

Lily C Kumbani

Malawi

Su-Chen Kuo

Taiwan

Zehra Kurdoglu

Turkey

Juan Kusanovic

USA

Raman Kutty

India

Anne Stine Kvehaugen

Norway

Linda Kvist

Sweden

Harm Jan Kwikkel

Netherlands

Elisabeth Kylberg

Sweden

Catherine Kyobutungi

Kenya

Elisa Lacerda

Brazil

Eve Lackritz

USA

Ching Tat Lai

Australia

Hugh Simon Hung San Lam

Hong Kong

Steven Lamm

USA

Jens Langhoff-Roos

Denmark

Sophia Lannon

USA

Hermann Lanou

Burkina Faso

Annunziata Lapolla

Italy

Martha Lappas

Australia 
Elysia Larson

USA

Anne-Marie Laslett

Australia

Laura Lauria

Italy

Tina Lavender

UK

Denise Lawler

Ireland

Beverley Lawton

New Zealand

Liana Leach

Australia

Nicky Leap

Australia

Meng-Chih Lee

Taiwan

Sue Lee

Thailand

Ana Leslie

Brazil

Felicia Lester

USA

Ky Leung

Hong Kong

Adrienne Levay

Canada

Sarah Lewis

UK

Changwei Li

USA

Ching-Chiang Lin

Taiwan

Helena Lindgren

Sweden

Robert Liston

Canada

Siu-Wai Lit

Hong Kong

James Litch

USA

Ching-Ming Liu

Taiwan
Tsz Kin Lo

China

Robert Locke

USA

Terhi Lohela

Finland

Elin Bjørge Løken

Norway

Carl Lombard

South Africa

Mary Longworth

UK

Yolanda Lopez

Spain

Jorge Lopez-Camelo

Argentina

Fred Lotgering

Netherlands

Jimmy Chun Yu Louie

Australia

Svetla Loukanova

Germany

Mona Loutfy

Canada

Julia Lowe

Canada

Richard Lowe

USA

Miha Lucovnik

Slovenia

Mirjam Lukasse

Norway

Pisake Lumbiganon

Thailand

Ingela Lundgren

Sweden

Karsten Lunze

USA

Zhong-Cheng Luo

Canada

Angela Lupattelli

Norway

Jennifer Lutomski

Ireland
Courtney Lynch

USA

Nanna Maaløe

Denmark

Karen Mackinnon

Canada

Mohsen Maddah

Iran

Moke Magoma

Tanzania

Michael Johnson Mahande

Tanzania

Qun Mai

Australia

Abdulkarim Mairiga

Nigeria

Emma Louise Malchau Carlsen

Denmark

Maili Malin

Finland

Caroline Maltepe

Canada

Albert Manasyan

Zambia

Justin Mandala

USA

Jeevan Marasinghe

Sri Lanka

Dannette Marie

UK

Lena B Martensson

Sweden

Helene Martin

Switzerland

Rocio Martin

Netherlands

Wellington Martins

Brazil

Samantha Mascelli

Italy

Gileard Masenga

Tanzania

Edwin Massey

UK 
Matthews Mathai

Switzerland

Ilan Matok

Israel

Hiroshi Matsushita

Japan

Anne Matthews

Ireland

Robyn Maude

New Zealand

Godfrey Michael Mbaruku

Tanzania

Columba K Mbekenga

Tanzania

Judith Mcara-Couper

New Zealand

Emma Mccall

UK

Janya Mccalman

Australia

Elizabeth Mccarthy

Australia

Anthony Desmond Mccarthy

Argentina

Elizabeth Mcclure

USA

Colin Mccord

UK

Christine Mccourt

UK

Ellie Mcdonald

Australia

Susan Mcdonald

Australia

Ted Mcdonald

Canada

Alison Mcfadden

UK

Rose Mcgready

Thailand

Tania Mcintosh

UK

Meredith Mcintyre

Australia
Helen Mclachlan

Australia

Tarek Meguid

Malawi

Tarek Meguid

Namibia

Azar Mehrabadi

Canada

Yared Mekonnen

Ethiopia

N Melamed

Israel

Antonella Mencacci

Italy

Sotero Mengue

Brazil

Ramkumar Menon

USA

Fiona Mensah

UK

Astrid Merkx

Netherlands

Abi Merriel

UK

Amy Metcalfe

Canada

Carla Meurk

Australia

Jill Mhyre

USA

Antonina Mikocka-Walus

UK

Annie Mills

Australia

Ghazala Mir

UK

Jezid Miranda

USA

Shiva Raj Mishra

Nepal

Malgorzata Miszkurka

Canada

Bryan Mitchell

Canada
Prasanna Mithra

India

Ankita Mittal

India

Blandina Theophil Mmbaga

Norway

D Paul Moberg

USA

Vernon Mochache

Kenya

Ingrid Mogren

Sweden

Ben Mol

Afghanistan

Mitike Molla

Ethiopia

Mark Monahann

Ireland

Rob Mooij

Netherlands

Tiffany Moore Simas

USA

Tahereh Moradi

Sweden

Imran Morhason-Bello

Nigeria

Rafael Moroni

Brazil

Pamela Morrison

UK

Michelle Mottola

Canada

Cheryl Moyer

USA

Mwifadhi Mrisho

Tanzania

Projestine Muganyizi

Tanzania

Ashar Muhammad Malik

Pakistan

Peter Mukasa

Uganda

Kathryn Muldoon

Ireland 
Luke Mullany

USA

Sunni Mumford

USA

Zubia Mumtaz

Canada

Sylvia Murphy Tighe

Ireland

Andrew James Murray

UK

Jonah Musa

Nigeria

Adamson Muula

Malawi

Beatrice Mwagomba

Malawi

Ipyana Mwampagatwa

Tanzania

Emanuel Mwendo

Tanzania

Elizabeth Nabiwemba

Uganda

Harriet Nabudere

Uganda

Mona Nabulsi

Lebanon

Manisha Nair

UK

Hacer Nalbant

Turkey

Jane Namasasu

Malawi

Bejoy Nambiar

Malawi

Kavita Nanda

USA

Jolly Nankunda

Uganda

Rahel Nardos

USA

Natasha Nassar

Australia

Dipty Nawal

India
Juliet Ndibazza

Uganda

Ellen Nelissen

Tanzania

Sutapa Neogi

India

Gustavo Neppelenbroek

Brazil

Robin Nesbitt

Germany

John Newell

Ireland

James Newham

UK

Shu-Kay Angus Ng

Australia

Caroline Nguluwe

Malawi

Thinh Nguyen

Australia

Susan Niermeyer

USA

Marianne Nieuwenhuijze

Netherlands

Bahareh Nikooyeh

Iran

Christina Nilsson

Sweden

Somashekhar Nimbalkar India

I Nippert

Germany

Tanya Nippita

Australia

Yoshihiro Nishida

Japan

Kaijun Niu

Japan

Elie Nkwabong

Cameroon

Roseli M Y Nomura

Brazil

Randa Nooh

Saudi Arabia
Shane Norris

South Africa

Angelo Nyamtema

Tanzania

Kerstin H Nyqvist

Sweden

Anicet Nzabonimpa

Rwanda

Jeremy Oats

Australia

Colm Oboyle

Ireland

Beverley O'Brien

Canada

Pat O'Brien

UK

Michel Odent

UK

Ju Lee Oei

Australia

Olanma Ogbuehi

UK

Kerry-Ann O'Grady

Australia

Tinuade Ogunlesi

Nigeria

Magdalena Ohaja

Ireland

Felix Okah

USA

Okechukwu Angelis Okezie

Botswana

Ellinor Olander

UK

Oladapo Olayemi

Nigeria

Joann O'Leary

USA

Purevdorj Olkhanud

USA

Per Olofsson

Sweden

Christine Olson

USA 
David Olson

Canada

Bolajoko O. Olusanya

Nigeria

Ayodeji Oluwole

Nigeria

Siti Zawiah Omar

Malaysia

Sinead M. O'Neill

Ireland

Maricianah Atieno Onono

Kenya

Sharad Onta

Nepal

Marieke Oostvogels

Netherlands

Carlos Ortega González

Mexico

Alfred Osoti

Kenya

David Osrin

UK

Eugene Oteng-Ntim

UK

Ellis Owusu-Dabo

Ghana

Semra Ozdemir

Singapore

Carmen Pace

Australia

Julie Pallant

Australia

Chandrakant Pandav

India

Sarita Panday

Nepal

Damaru Paneru

Nepal

Eleni Papadopoulou

Norway

Theodore Papaioannou

Greece

Shantini Paranjothy

UK
Min Hae Park

UK

Angela Pascall
UK
Dharmintra Pasupathy
UK
Jillian Patterson
Australia
Deepak Paudel
Nepal
Deborah Payne
New Zealand
Christopher Pell
Netherlands

Andrea Barnabas Pembe

Tanzania

Suzanne Penfold

UK

Suzanne Penfold

Slovakia

Teuila Percival

New Zealand

Gloria Pérez

Spain

Susan Perlen

Australia

Amber Peterman

USA

Wendy Peterson

Canada

Stamatios Petousis

Greece

Constanze Pfeiffer

Switzerland

Sharon Phelan

USA

Suparat Phuanukoonnon

Papua New Guinea

Maria Piancino

Italy

Jashvant Poeran

Netherlands

Paras K Pokharel

Nepal
Wendy Pollock

Australia

Nancy Poole

Canada

Maree Porter

Australia

Malcolm Potts

USA

Jennifer Powers

Australia

Robert Powers

USA

Ndola Prata

USA

Holly Priddis

Australia

Danielle Kaye Prime

Australia

Samantha Prosser

Australia

Audrey Prost

UK

Wendy Prudhomme O'Meara

Kenya

Roopa Ps

India

Giuseppe Puccio

Italy

Caroline Pukall

Canada

Eero Pukkala

Finland

Shuby Puthussery

UK

Xu Qian

China

Liqian Qiu

China

Parvin Rahnama

Iran

Sari Räisänen

Finland

Augustine Rajakumar

USA 
Tonse Raju

USA

Meenakshi Ramphul

Ireland

Heribert Ramroth

Germany

Deborah Randall

Australia

Bharat Randive

India

Svein Rasmussen

Norway

Erica Rauff

USA

Anita Ravelli

Netherlands

Joanna Raven

UK

Sundari Ravindran

India

Joel Ray

Canada

Angela Reichelt

Brazil

Joerg Reichert

Germany

Cavan Reilly

USA

Liv Merete Reinar

Norway

Kristina Martha Renault

Denmark

Udo Reulbach

Ireland

Annette Reuss

Germany

Grazzia Rey

Uruguay

Richard Rheingans

USA

Kalisa Richard

Rwanda

Carol Richardson

UK
Anne Rifkin-Graboi

Singapore

Machteld Rijken

Netherlands

Marcus Rijken

Thailand

Marcus Rijken

Netherlands

Marlies Rijnders

Netherlands

Monica Rittler

Argentina

Demetrios Rizos

Greece

Julie Robitaille

Canada

Dianne Rodger

Australia

Anna Joy Rogers

USA

Cheong Rae Roh

Korea South

Liliam Cristine Rolo Paiato

Brazil

Ted Rosales

Canada

Alan Rothberg

South Africa

Ingrid Rowlands

Australia

Jenny Ruducha

USA

Stephen Rulisa

Rwanda

Alice Rumbold

Australia

Audrey Saftlas

USA

Joanne Said

Australia

Evelyn Sakeah

Ghana

Kabiru Salami

Nigeria
Sarah Saleem

Pakistan

Raed Salim

Israel

Jenny Salmon

New Zealand

Birgitta Salomonsson

Sweden

Jacob Sandberg

Sweden

Julia Sanders

UK

Eduardo Santana

Brazil

Nejc Sarabon

Slovenia

Carolyn Sargent

USA

Narendra Nath Sarkar

India

Benn Sartorius

South Africa

Sadath Sayeed

USA

Jules Schagen Van Leeuwen

Netherlands

Ashley Schempf

USA

Virginia Schmied

Australia

Georg Schmolzer

Canada

Christiane Schwarz

Germany

Jane Scott

Australia

Marie Scully

UK

Anna Seale

UK

Hannah Motshedisi Sebitloane

South Africa

Kerem Doga Seckin

Turkey 
Innocent Semali

Tanzania

Tetine Sentell

USA

Karen Sepucha

USA

Marie Seraphin

USA

Elisabeth Severinsson

Norway

Prakeshkumar Shah

Canada

Antonia Shand

Australia

Caitlin Shannon

USA

Sheetal Sharma

UK

Sudesh Raj Sharma

Nepal

Alison Shaw

UK

Zoe Sheppard

UK

Ashalatha Shetty

UK

Eiji Shibata

Japan

Shashikant Sholapurkar

UK

Binjwala Shrestha

Nepal

Alexis Shub

Australia

Joseph Shumway

USA

Crystal Silva

USA

Robert Silver

USA

Kyra Sim

Australia

Leickness Chisamu Simbayi

South Africa
Melissa Simon

USA

Nigel Simpson

UK

Gunjan Singh

United Arab Emirates

Kavita Singh

USA

Rameet Singh

USA

Sodiomon Bienvenu Sirima

Burkina Faso

Deborah Sitrin

USA

Valeria Skafida

UK

Nancy L Sloan

USA

Maria Small

USA

Rhonda Small

Australia

Debbie Smith

UK

Jeffrey Michael Smith

USA

Julie Smith

Australia

Valerie Smith

Ireland

John Smulian

USA

Erna Snelgrove-Clarke

Canada

Jonathan Snowden

USA

Malin Soederberg

Sweden

Andrea Solnes Miltenburg

Netherlands

Priya Soma-Pillay

South Africa

Ingvil Krarup Sørbye

Norway
Alexandros Sotiriadis

UK

Carina Sparud-Lundin

Sweden

Georgina Spies

South Africa

Henri Spronk

Netherlands

Chandrashekhar Sreeramareddy

Nepal

Anke Steckelberg

Germany

M Steinhoff

USA

Jelle Stekelenburg

Netherlands

Olof Stephansson

Sweden

Alexandre Stephens

Australia

Joanna Stewart

New Zealand

Mary Stewart

UK

Zoe Stewart

UK

Sarah Stock

UK

Kathrin Stoll

Germany

Hege Therese Størksen

Norway

Zhixian Sui

Australia

Sa'Adatu Sule

Nigeria

Bo Sun

China

Johanne Sundby

Norway

Fernanda G C Surita

Brazil

Elisabeth Svensson

Sweden 
Elizabeth Taglauer

Ireland

Mizuki Takegata

Japan

Yin On Yvonne Tam

USA

Berhanu Tameru

USA

Fangbiao Tao

China

Belaynew Taye

Ethiopia

Lee Taylor

Australia

Kate Teela

USA

Gerben Ter Riet

Netherlands

Christian Teter

USA

Li Thies-Lagergren

Sweden

Gill Thomson

UK

James Tielsch

USA

Christiana Titaley

Indonesia

Deirdre Tobias

USA

Rachel Tolhurst

UK

Gabriele Tonni

Italy

Jocelyn Toohill

Australia

Maria Regina Torloni

Brazil

Christopher Torrens

UK

Siranda Torvaldsen

Australia

Clare Tower

UK
Thach Tran

Viet Nam

Naomi Trengove

Australia

Dominic Trepel

UK

Vandana Tripathi

USA

Amy Tsui

USA

Sandy Tubeuf

UK

Ozge Tuncalp

USA

Deborah Turnbull

Australia

Nana A. Y. Twum-Danso

USA

Zia Ul-Haq

UK

Odi' Ugochukwu Joannes Umeora

Nigeria

Aniefiok Umoiyoho

Nigeria

Julia Unterscheider

Australia

Michele Upvall

USA

Michael Urban

South Africa

Julio Urrutia

Chile

Ihab Usta

Lebanon

Michele Usuelli

Italy

Bettina Utz

Belgium

Marja Vääräsmäki

Finland

Edi Vaisbuch

USA

Christine Valente

UK
Frédérique Vallières

Ireland

Jodie Valpied

Australia

Heleen Van Beekhuizen

Netherlands

Rob Van Dam

Singapore

Peter Van De Ven

Netherlands

Jef Van Den Ende

Belgium

Albertine Van Der Does

South Africa

Frits Van Der Haar

USA

Jacoba Van Der Kooy

Netherlands

Carla Van Der Wijden

Netherlands

Nickie Van Der Wulp

Netherlands

Paul Van Kesteren

Netherlands

An-Sofie Van Parys

Belgium

Lenie Van Rossem

Netherlands

Vicki Van Wagner

Canada

Christiaan Van Woerden

Netherlands

Jeroen Vanderhoeven

USA

Linda Vanotoo

Ghana

Paula Vargas Innocenti

Chile

Manu Vatish

UK

Saraswathi Vedam

Canada

Katri Vehvilainen-Julkunen

Finland 
Marjolein Verhart

Netherlands

Stefan Verlohren

Germany

Tienke Vermeiden

Ethiopia

Rose Victor

Tanzania

Francesco Vierucci

Italy

Jenny Vieveen

Netherlands

Simone Vigod

Canada

Anne Britt Vika Nilsen

Norway

Divya Vohra

USA

Frauke Von Versen-Höynck

Germany

Franziska Wadephul

UK

Yohannes Dibaba Wado

Ethiopia

Lorraine Walker

USA

Dilys Walker

USA

L. Lewis Wall

USA

Julia Walsh

USA

Yuping Wang

USA

Carol Wanjira

Kenya

Rhoda Wanyenze

Uganda

Nicola Wardrop

UK

Lucie Warren

UK

Arjumand Warsy

Saudi Arabia
Lyndsey Watson

Australia

Andrew Weeks

UK

Mary Nell Wegner

USA

Carolyn Weiniger

Israel

Diana Wellesley

UK

Barbara Welles-Nystrom

USA

Michael Wells

Sweden

Shi Wu Wen

Canada

Heather Whitford

UK

Eva Wiberg-Itzel

Sweden

Therese Wiegers

Netherlands

Hanne Benedicte Wielandt

Denmark

Ingela Wiklund

Sweden

Carol Wilkins

UK

Chris Wilkinson

Australia

Shelley Wilkinson

Australia

Jane Willcox

Australia

Calistus Wilunda

Italy

Martha Wingate

USA

Brita Askeland Winje

Norway

Katherine Wisner

USA

Cynthia Wong

USA
Cindy Woods

Australia

Abebaw Worku

Ethiopia

Francesca Wuytack

Ireland

Karen Wynter

Australia

Haipeng Xiao

China

Xu Xiong

USA

Mohammad Yakoob

USA

Mohsin Yakub

USA

Eileen Yam

USA

Gavin Yamey

USA

Junta Yanai

Japan

Zhenyu Yang

China

Hung-Wen Yeh

USA

Jane Yelland

Australia

Tegbar Yigzaw

Ethiopia

Susanha Yimyam

Thailand

Koyo Yoshida

Japan

David Young

Canada

Sera Young

USA

Shamsa Zafar

Pakistan

Partamin Zainullah

Afghanistan

Vincenzo Zanardo

Italy 
Giovanni Zanconato

Italy

Phyllis Zelkowitz

Canada

Lubo Zhang

USA
Jing Zheng

USA

Mariusz Zimmer

Poland

Marcelo Zubaran Goldani

Brazil

\section{Luisa Zuccolo}

UK

Rose Zulliger

USA

Prisca Ac Zwanikken

Netherlands 